\title{
ARTICLE
}

\section{Development of analysis method for airborne radiation monitoring using the inverse problem solutions}

\author{
Miyuki Sasaki*, Azusa Ishizaki and Yukihisa Sanada \\ Fukushima Environmental Safety Center, Japan Atomic Energy Agency, 45-169 Sukakeba, Kaibama-aza, Haramachi, Minamisoma, \\ Fukushima, 975-0036, Japan
}

\begin{abstract}
Following the accident at the Fukushima Daiichi Nuclear Power Plant, some kind of unmanned aerial vehicles (UAVs) have been used to collect environmental radiation data. The Japan Atomic Energy Agency has been developed methods for analyzing radiation measurements taken by a small multirotor UAV. In the conventional method, count rate was converted to the air dose rate using a simple parameter. Therefore, such a conversion of the air dose rate using the conventional method is difficult in an area where the distribution of air dose rate is heterogeneous, or the terrain is complex. The goal of this study was to apply the inverse radiation method, which is used in medical applications such as positron emission tomography, for environmental radiation measurement. Basic algorithm was established for conversion from count rate to air dose rate in considering of topographic data. The algorithm was applied to real radiation data obtained by a UAV, and its accuracy was evaluated by comparing that data with ground measurement data. As compared with conventional methods, our algorithm was demonstrated to achieve a more accurate estimation of the air dose rate on the ground.
\end{abstract}

Keywords: $U A V$; remote radiation measurement; terrain correction; inverse radiation problem method; Fukushima Daiichi Nuclear Power Plant accident

\section{Introduction}

After the accident at Fukushima Daiichi Nuclear Power Station (FDNPS), the manned helicopter and the unmanned aerial vehicle (UAV), which is R-MAX G1 by YAMAHA Co., Ltd. originally developed for spraying pesticides, was have been used for aerial radiation measurement $[1,2]$. The state-of-the-art multirotor UAV can be used to take measurements of environmental radiation. UAVs are inexpensive, compact, and easy to operate. The Japan Atomic Energy Agency has been investigated the use of an UAV for measuring the distribution of air dose rates in the environment [3]. The method for transmitting the detector data to the ground is based on the following assumptions; 1) The ambient dose rate at $1 \mathrm{~m}$ on the ground (to be referred to as "air dose rate" in the following) is constant. 2) The terrain model is flat, which uses the flat surface model (FSM). 3) The count rates are exponentially correlated with altitude of detector. It is challenging to convert the air dose rate to a precise ground value at a measurement location where the distribution of air dose rate at $1 \mathrm{~m}$ agl. is not uniform or terrain is complex. The goal of this study was to establish a new analytical method based on the inverse radiation problem used in medical applications, including positron emission tomography (PET). The method of maximum-likelihood expectation-maximization (ML-EM) [4, 5] was selected for conversion from the count rate to the air dose rate. ML-EM was applied to real data acquired by an UAV, and its performance was evaluated by comparing the results of aerial measurements with that of ground measurements.

\section{Materials and methods}

\subsection{UAV and radiation measurement system}

We used a commercial UAV system (3D Robotix Inc., California, USA). Figure 1 shows a schematic of the UAV. The UAV had dimensions of 990 (width) $\times 990$ (depth) $\times 250$ (height) $\mathrm{mm}^{3}$, a weight of $5 \mathrm{~kg}$ (including detector and battery), and a maximum flight time of approximately $10 \mathrm{~min}$. An emergency stop function and propeller guard were fitted to the system for safety. The position of the UAV was tracked by a Global Positioning System (GPS), and an operator controlled the flight path, speed, and altitude of UAV.

*Corresponding author. Email: sasaki.miyuki@jaea.go.jp 


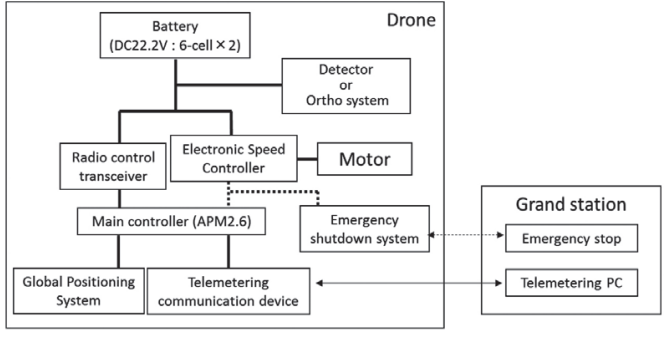

Figure 1. Schematic diagram of the UAV.

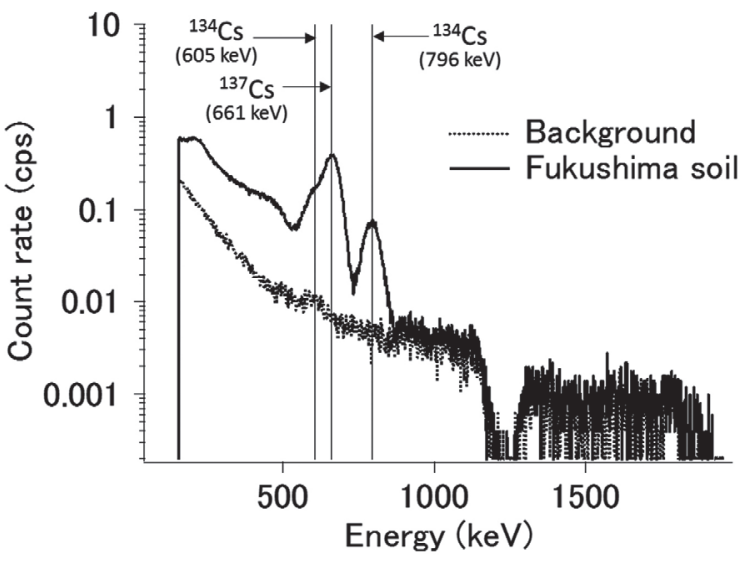

Figure 2. Example of the gamma-ray spectrum from the GAGG scintillator.

Radiation was detected by a $2 \times 2 \times 2 \mathrm{~cm}^{3}$ gadolinium aluminum gallium garnet scintillation detector (GAGG detector: Furukawa Co, Ltd, Tokyo, Japan). The detection of gamma-ray was ranged from 0.1 to 2.0 $\mathrm{MeV}$. The energy resolution (Full width at half maximum) at $662 \mathrm{keV}$ was less than $9.8 \%$. The example of gamma-ray spectrum is shown in Figure 2. The dotted line shows the background spectrum, and the solid line shows the spectrum of soil contaminated by radiocesium $\left({ }^{134} \mathrm{Cs}\right.$ and $\left.{ }^{137} \mathrm{Cs}\right)$ sampled at Fukushima prefecture. The data of gamma-ray spectrum and GPS were acquired every 3 seconds.

Ground measurements were performed using a handheld cesium iodide (CsI) scintillation detector to record air dose rates $1 \mathrm{~m}$ above the ground level (agl). In addition, ortho photo images were taken to construct a digital surface model. Aerial and ground radiation measurement was conducted in Fukushima.

\subsection{Analysis methods}

\subsubsection{Conventional method (FSM)}

Exponential correlation of count rate with altitude of detector was first conducted using the FSM as the source model [6]. In a calibration flight, the count rate was obtained at settled altitude in a relatively flat area. The effective radiation attenuation factor was calculated from the altitude and the count rate data obtained in the calibration flight. This yielded a conversion to air dose rate by using the average of the measurement values.

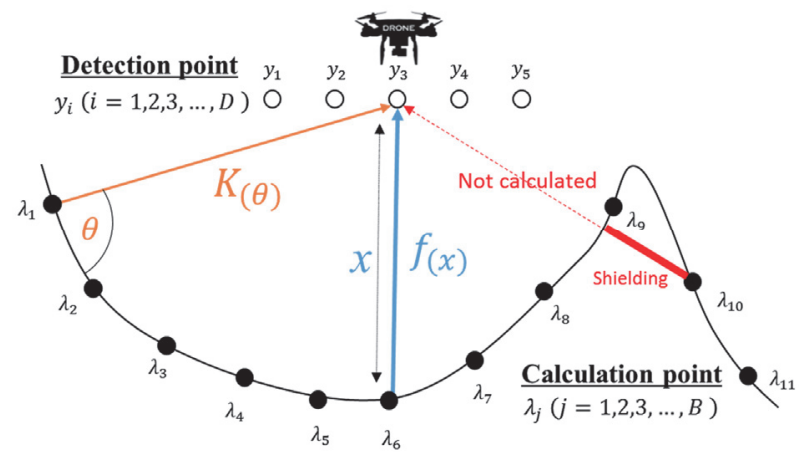

Figure 3. Calculation image of ML-EM method.

\subsubsection{Theory of ML-EM method}

The ML-EM method is an inverse radiation method that is used for image reconstruction in PET scans. The calculation image of ML-EM method is shown in Figure 3. Theory of the method is as follows;

$$
\lambda_{j}^{k+1}=\frac{\lambda_{j}^{k}}{\sum_{i=1}^{D} C_{i j}} \sum_{i=1}^{D} \frac{y_{i} C_{i j}}{\sum_{j=1}^{B} \lambda_{j}^{k} C_{i j}}
$$

where $k$ is the number of iterations, $j$ is the calculation position on the ground, $B$ is the total number of $j, i$ is the detection point position, and $D$ is the total number of $i$.

$\lambda_{j}^{k}$ and $\lambda_{j}^{k+1}$ correspond to $k$ and $k+1$ ground calculated values, respectively. $y_{i}$ is the measured value and $C_{i j}$ is defined the parameter that is including the attenuation coefficient and detector efficiency. The $C_{i j}$ was calculated using the particle and heavy ion transport code system (PHITS) [7]. $C_{i j}$ is expressed as follows;

$$
\begin{aligned}
C_{i j}=K_{(\theta)} \times f_{(x)} \\
K_{(\theta)}=y_{0}+B \exp \left[-\left(\frac{\theta-x_{0}}{\sigma}\right)^{2}\right] \\
\quad \begin{array}{l}
f_{(x)}=x^{-\mu_{1}}: x \leq 200 \\
f_{(x)}=\frac{200^{-\mu_{1}}}{\exp \left(-200 \mu_{2}\right)} \times \exp \left(-\mu_{2} x\right): x>200
\end{array}
\end{aligned}
$$

where $K(\theta)$ is the attenuation coefficient of the angle between $j$ and $i$. PHITS results for photon attenuation by angle $(\theta)$ of line of $i$ and $j$ against soil surface line is shown in Figure 4. The contribution ratio of scattered ray to the detector decreases when $\theta$ is small. The value of $K(\theta)$ was obtained by Gaussian approximation as shown by Eq. (3).

The $f(x)$ is the attenuation coefficient of the distance from $j$ to $i$. PHITS results for photon attenuation by distance from the point source $\left({ }^{137} \mathrm{Cs},{ }^{40} \mathrm{~K}\right.$ and $\left.{ }^{208} \mathrm{Tl}\right)$ are shown in Figure 5. The approximation $f(x)$ is expressed by Eqs. (4) and (5) because approximate expressions of $f(x)$ are different depending on distance from $j$ to $x$. As 


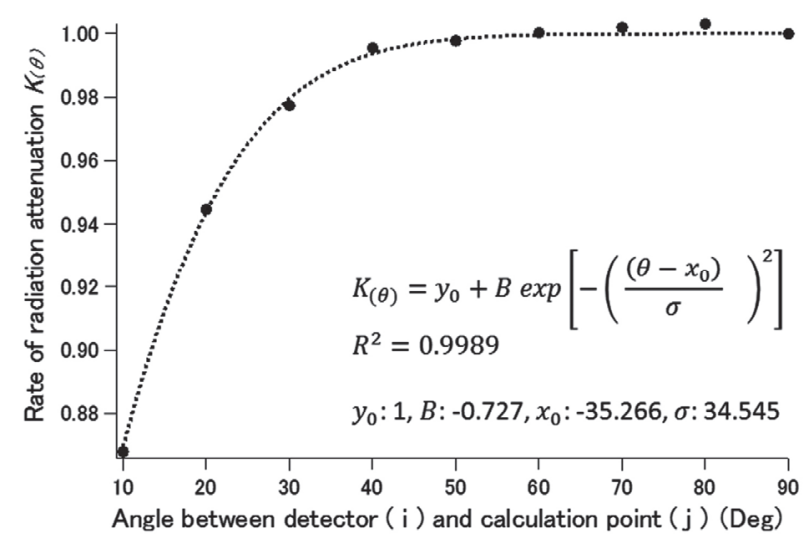

Figure 4. Attenuation factors calculated of angle by PHITS.

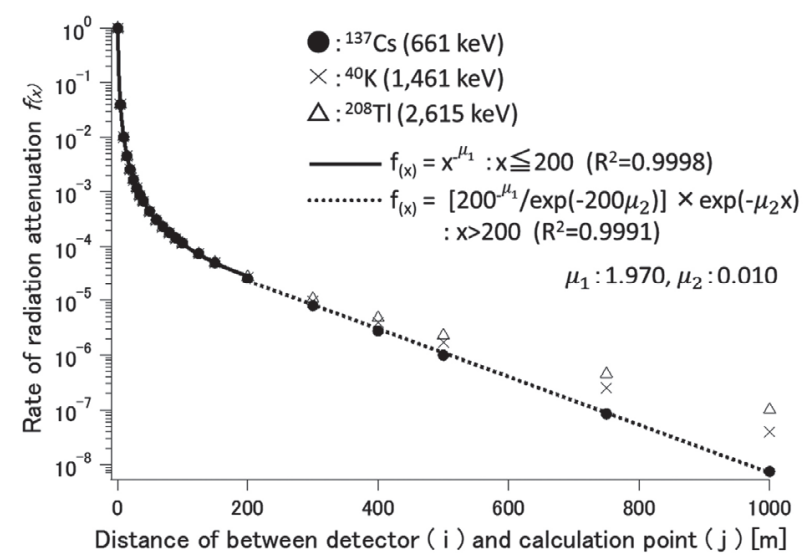

Figure 5. Attenuation factors calculated of distance by PHITS.

shown in Figure 5, power approximation Eq. (4) was derived at distance up to $200 \mathrm{~m}$ and exponential approximation Eq. (5) was derived at distance greater than $200 \mathrm{~m}$. Calculation results of $f(x)$ at three energies when calculating $x>500$ was almost agree. In this study, conversion of ML-EM method used attenuation factor with the energy of $662 \mathrm{keV}{ }^{137} \mathrm{Cs}$ point source.

\subsubsection{Evaluation of accuracy}

The difference between the ground value and the converted value of the UAV data was quantified using the normalized mean square error (NMSE) method. This method is expressed as follows;

$$
N M S E=\frac{\sum_{i=1}^{C}\left(G_{i}-V_{i}\right)^{2}}{\sum_{i=1}^{C} G_{i}^{2}}
$$

where $C$ is the total number of data points, $G$ is the ground value, and $V$ is the converted value.

\section{Results and discussion}

\subsection{Benchmark test}

For benchmark testing of the ML-EM method, we obtained measurement data from a point radiation source

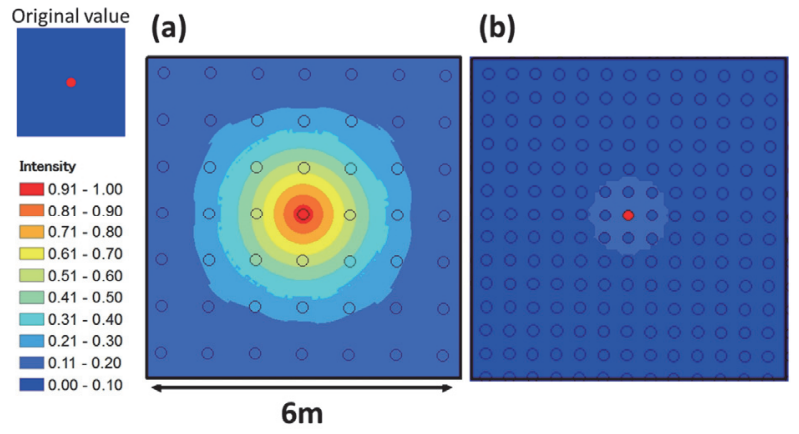

Figure 6. Comparison of normalized dose rate maps produced by conventional method and ML-EM method $(k=1000)$ in benchmark test. The circles $(\bigcirc)$ in (a) are measurement points and those in (b) are calculation points.

$\left({ }^{137} \mathrm{Cs}: 10 \mathrm{MBq}\right)$ placed in the center of the laboratory $(6$ $\mathrm{m} \times 6 \mathrm{~m})$. Count rate at $1 \mathrm{~m}$ agl. using a GAGG scintillation detector was obtained using a $1 \mathrm{~m}$ mesh. The data was converted to air dose rate at $5 \mathrm{~cm}$ agl. using the conventional method and ML-EM method. In the conventional method, the air attenuation factor was derived using the theoretical value calculated by PHITS and shown in Figure 4. In the ML-EM method, calculation points were calculated within $12 \mathrm{~m} \times 12 \mathrm{~m}$ area with a $50 \mathrm{~cm}$ mesh. A contour map of air dose rates was plotted using the Kriging method in ArcGIS software (ESRI Co., Ltd., California, USA). The benchmark test conversion from the conventional method is shown in Figure 6 (a), and the conversion from the ML-EM method is shown in Figure $6(\mathbf{b})$.

$\lambda_{j}^{0}$ was set to measurement average value in Figure 6

(b). The conversion values were normalized by division of maximum value. The true value is that the central point is 1 and other points are 0. The NMSE of converted values by the conventional method and the ML-EM method $(k=1000)$ were 3.00 and 0.72 , respectively. It is possible to estimate the size of source more accurately than the conventional method when the position of measurement is far from the source. The convergence of calculations became faster according as $\lambda_{j}^{0}$ is closer to the original value.

\subsection{Field tests}

\subsubsection{Flat area}

Radiation data was measured by a UAV in a flat area in Fukushima prefecture. The measurement area, altitude, speed, and flight line spacing were $50 \times 150 \mathrm{~m}^{2}, 10 \mathrm{~m}$ agl, $2 \mathrm{~m} \mathrm{~s}^{-1}$, and $10 \mathrm{~m}$, respectively. The ground measurement value at $1 \mathrm{~m}$ agl taken by the handheld survey meter is shown in Figure 7 (a). The airborne radiation measurement results of conversion to $1 \mathrm{~m} \mathrm{agl}$ by the conventional method and ML-EM method are shown in Figures 7 (b) and (c), respectively. The results of ratio to the ground value between the conventional method and ML-EM method are shown in Figures 7 (d) and (e), respectively. NMSE of the calculated value $(\lambda)$ to the ground value became the minimum when the 


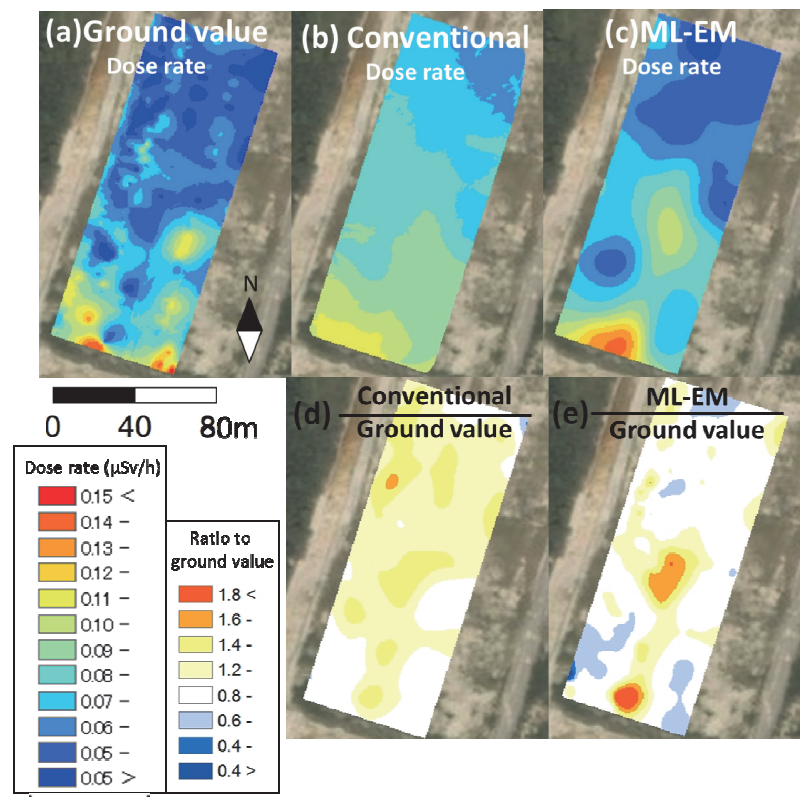

Figure 7. Comparison of radiation values measured by UAV and by ground measurement in flat area.
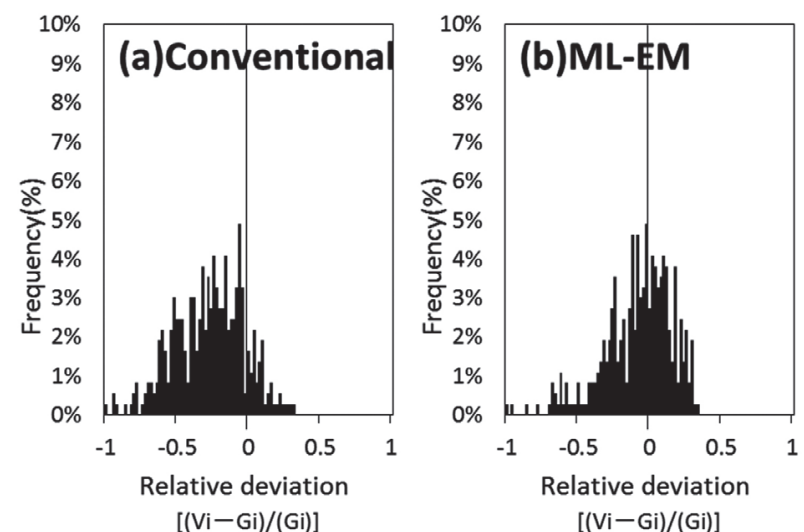

Figure 8. Relative deviation of conversion results in flat area.

number of calculations was 14 times $(k=14)$. Calculation iteration was important parameter in analyzing unknown result of a measurement. The problem of calculation iteration was described in section 3.3. The air dose rate was higher at the south side than that at the north side within area. Relative deviation between the ground value and the converted value are shown in Figure 8. The NMSE value of converted by the conventional method was 0.11 , compared with 0.07 for conversion using the ML-EM method. The results indicated that the ML-EM method was an effective approach to map the air dose rate distribution more accurately than the conventional method.

\subsubsection{Complex terrain area}

General residential areas (including houses, paddy fields, and forests) were selected as an example of more complex terrain. The measurement area, altitude, speed, and flight line spacing were $90 \times 110 \mathrm{~m}^{2}, 10-50 \mathrm{~m} \mathrm{agl}, 2$ $\mathrm{m} \mathrm{s}^{-1}$, and $10 \mathrm{~m}$, respectively. The ground measurement

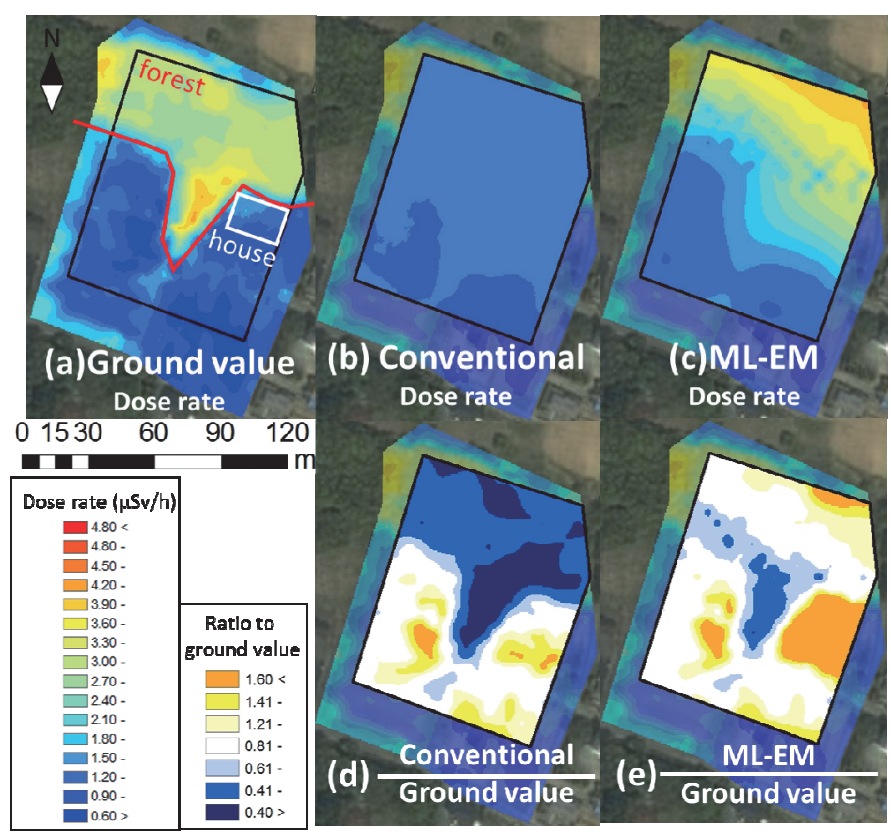

Figure 9. Conversion result of radiation measurement values by micro UAV and ground measurement in complex terrain area.
(a)Conventional

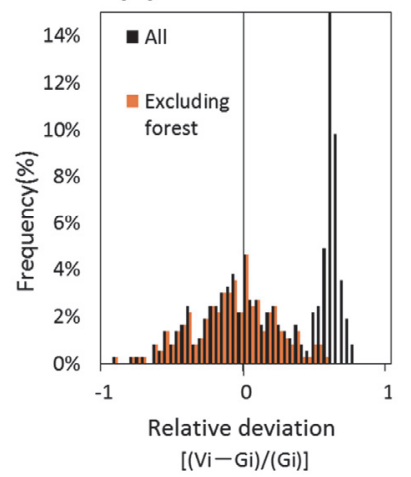

(b)ML-EM

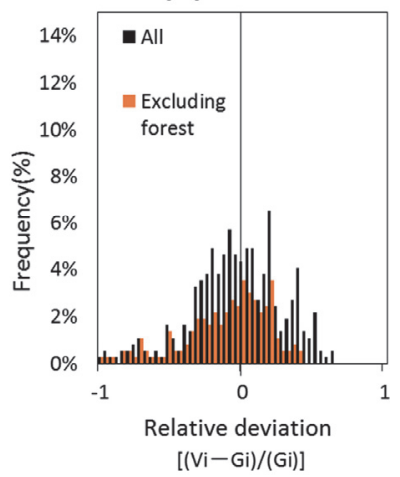

Figure 10. Relative deviation of conversion results in complex terrain area.

value at $1 \mathrm{~m}$ agl. obtained by the handheld meter is shown in Figure 9 (a). The results of conversion to $1 \mathrm{~m}$ agl. by the conventional method and ML-EM method are shown in Figures 9 (b) and (c), respectively. The results of ratio to the ground value between the conventional method and ML-EM method are shown in Figures 9 (d) and (e), respectively. The air dose rate in forest area was relatively high compared to entire study site. In contrast, the air dose rate in south side was low because of decontamination works. The deviation between the ground value and the converted value are shown in Figure 10.

The NMSE value of conversion by the conventional method was 0.33 , compared with 0.13 for conversion using the ML-EM method. The Orange bar in Figure 10 is relative deviation of converted value in forest-free data. The double peak in Figure 10 (a) is due to forest. Relative deviation excluding forest area data was one peak. As can be seen from Figure 9, the conversion 

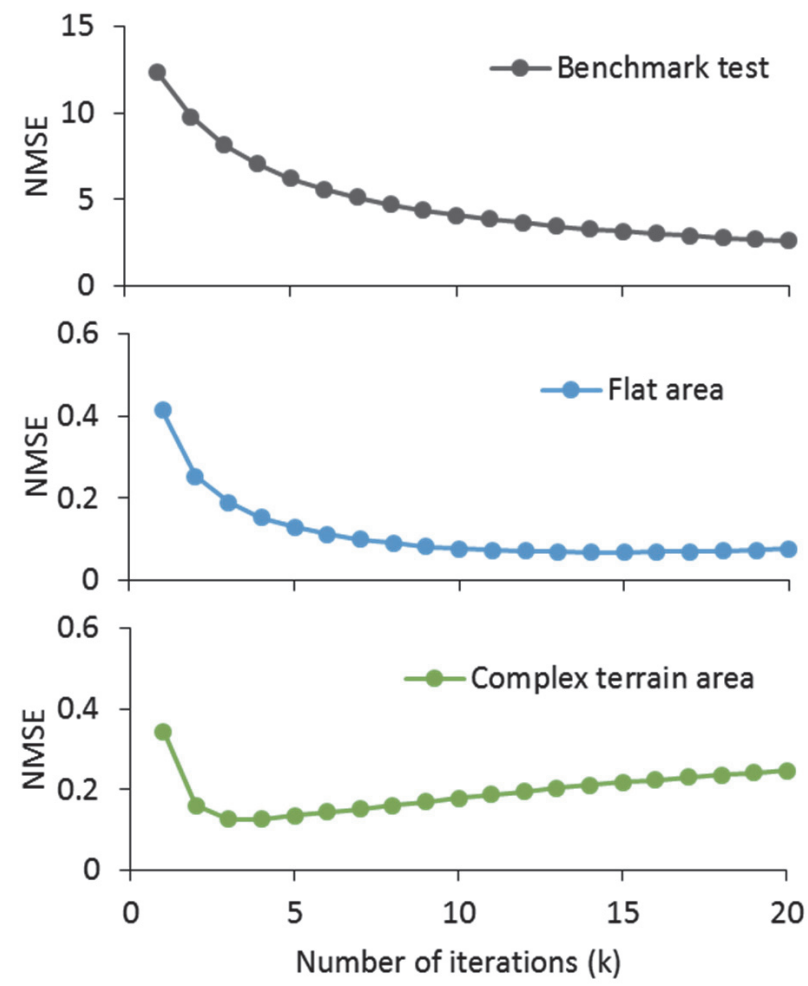

Figure 11. Relationship between iteration $(k)$ of ML-EM method and NMSE.

using the ML-EM method was not in agreement with the measurements taken by the handheld survey meter. It was attributed to attenuation of gamma-ray by trees not being considered in the ML-EM method. NMSE of the calculated value $(\lambda)$ to the ground value became the minimum when the number of calculations was 4 times $(k=4)$.

\subsection{Calculation iteration}

The relationship between the calculation iteration of ML-EM method and NMSE is shown in Figure 11. In the benchmark test, NMSE was converged toward 0 with increasing the number of iterations. This is a typical example at the case of application of ML-EM method. On the other hand, the NMSE between calculated and ground measurement value was large with increasing number of calculation in field data. Especially, increase in NMSE was remarkable in complex terrain area including forest. It is suggested that some parameters were not enough for on-site situation. Moreover, uncertainty of the efficiency of ML-EM method parameter $\left(C_{i j}\right)$, measurement error and noise may cause such an abnormal calculation. We will improve performance of this algorism by the addition of the parameter in future.

\section{Conclusions}

In this study, a novel conversion method for UAV radiation monitoring was proposed by an algorithm based on the ML-EM method. The proposed conversion method was demonstrated to map the air dose rate distribution more accurately than the conventional method. Furthermore, optimization of the algorithm is expected to allow the construction of detailed maps of air dose rate distribution. In future work, the ML-EM algorithm should be extended to allow for attenuation of gamma-ray by trees.

\section{References}

[1] Y. Sanada, T. Sugita, Y. Nishizawa, A. Kondo and T. Torii, The aerial radiation monitoring in Japan after the Fukushima Daiichi nuclear power plant accident, Prog. Nuc. Sci. Tech. 4 (2014), pp. 76-80.

[2] Y. Sanada and T. Torii, Aerial radiation monitoring around the Fukushima Dai-ichi Nuclear Power Plant using an unmanned helicopter, J. Environ. Radioact. 139 (2015), pp. 294-299.

[3] Y. Sanada, T. Orita and T. Tori, Temporal variation of dose rate distribution around the Fukushima Daiichi nuclear power station using unmanned helicopter, Appl. Radiat. Isot. 118 (2016), pp. 308-316

[4] L.A. Shepp and Y. Vardi, Maximum likelihood reconstruction for emission tomography, IEEE Trans. Med. Imaging. 1:2(1982), pp. 113-122.

[5] K. Lange and R. Carson, EM reconstruction algorithms for emission and transmission tomography, J. Compt. Assisted. Tomogr. 8 (1984), pp. 306-316.

[6] International Atomic Energy Agency, Guidelines for Radioelement Mapping Using Gamma Ray Spectrometry Data, IAEA TECDOC 1363, (2003).

[7] T. Sato, K. Niita, N. Matsuda, S. Hashimoto, Y. Iwamoto, S. Noda, T. Ogawa, H. Iwase, H. Nakashima, T. Fukahori, K. Okumura, T. Kai, S. Chiba, T. Furuta and L. Sihver, Particle and heavy ion transport code system PHITS, Version 2.52, J. Nucl. Sci. Technol. 50:9 (2013), pp. 913-923. 